\title{
Vibrations of a Timoshenko beam on an inertial foundation under a moving load
}

\author{
Magdalena Ataman ${ }^{1 *}$ \\ ${ }^{1}$ Warsaw University of Technology, Faculty of Civil Engineering, 16 Lech Kaczyński Str., 00-637 \\ Warsaw, Poland
}

\begin{abstract}
In the paper vibrations of the Timoshenko beam on an inertial foundation subjected to a moving force are discussed. Considered model of the inertial foundation is described by three parameters. They take into account elasticity, shear and inertia of the subgrade. In the literature such model of the subgrade is called Vlasov or Vlasov-Leontiev model. The Timoshenko beam is traversed by a concentrated load, moving with uniform speed. Response of the beam is found from the governing equations of motion of the problem. Problem of forced vibrations and problem of free vibrations of the beam are solved. Damping of the system is taken into consideration. Solution of the problem is illustrated by numerical example.
\end{abstract}

\section{Introduction}

The problem of vibration of beams and plates, resting on foundations and subjected to moving loads is closely related to the dynamics of bridges, roads, airfield and railway pavements $[1,2,3]$.

A mathematical model describing transverse vibrations of a beam, including the effects of rotatory inertia and shear deformations, was presented by Timoshenko [4, 5]. The model is described by two coupled partial, differential equations of motion with two independent variables: transverse displacement $w=w(x, t)$ and rotation of the beam cross section $\psi=\psi(x, t)$.

In the past the Timoshenko model was analysed by many authors, e.g. [6, 7]. Also the Timoshenko beam resting on a foundation was a subject of many works, as $[8,9,10,11]$, and others. Most often the subgrade is modelled as an elastic foundation and the analyses are based on the model of subgrade presented by Winkler [12], e.g. [13, 14]. However in practice the Winkler model cannot represent a continuous elastic medium. More adequate model for a large class of subgrade materials was proposed by Pasternak [15] and analysed e.g. in $[16,17]$. The Pasternak model consists of the Winkler foundation and includes shear interaction additionally, but does not take into account inertia of subsoil.

\footnotetext{
* Corresponding author: m.ataman@il.pw.edu.p1
} 
In the paper we consider the soil model which is defined by three parameters. They take into account elasticity, shear and inertia of subgrade. The Timoshenko beam resting on a such inertial foundation and subjected to a moving force is analysed.

Solution of forced vibrations and free vibrations along with numerical example are presented.

\section{Governing equations}

The equations of vibration of the beam, taking into account the effect of rotary inertia and shear distortion, were first reported by Timoshenko [4, 5]. Considering Timoshenko model, two coupled, partial differential equations of motion and boundary conditions, in the case of a simply supported beam, can be written in the following form:

$$
\begin{gathered}
E J \frac{\partial^{2} \psi}{\partial x^{2}}+\kappa G A\left(\frac{\partial w}{\partial x}-\psi\right)=\rho J \frac{\partial^{2} \psi}{\partial t^{2}}, \\
\kappa G A\left(\frac{\partial^{2} w}{\partial x^{2}}-\frac{\partial \psi}{\partial x}\right)+p=\rho A \frac{\partial^{2} w}{\partial t^{2}}, \\
w(0)=0, \quad \frac{\partial \psi(0)}{\partial x}=0, \quad w(l)=0, \quad \frac{\partial \psi(l)}{\partial x}=0,
\end{gathered}
$$

where:

$w-$ is the beam deflection,

$\psi-$ is the angle of rotation of the beam cross section,

$p=p(x, t)=q(x, t)-r(x, t)-$ is the external loading acting on the beam.

Elimination of $\psi$ from the equations of motion of Timoshenko beam yields differential equation with unknown deflection of the beam $w$

$$
\begin{gathered}
E J \frac{\partial^{4} w}{\partial x^{4}}+\rho A \frac{\partial^{2} w}{\partial t^{2}}-\rho J\left(1+\frac{E}{\kappa G}\right) \frac{\partial^{4} w}{\partial x^{2} \partial t^{2}}+\frac{\rho^{2} J}{\kappa G} \frac{\partial^{4} w}{\partial t^{4}}= \\
=p-\frac{E J}{\kappa G A} \frac{\partial^{2} p}{\partial x^{2}}+\frac{\rho J}{\kappa G A} \frac{\partial^{2} p}{\partial t^{2}}
\end{gathered}
$$

Taking into account reaction of the subgrade modelled as Vlasov foundation

$$
r(x, t)=k_{s} w(x, t)-G_{s} \frac{\partial^{2} w(x, t)}{\partial x^{2}}+m_{s} \frac{\partial^{2} w(x, t)}{\partial t^{2}},
$$

and the force moving with constant velocity $v, q(x, t)=P \delta(x-v t)$ equation (1) becomes 


$$
\begin{gathered}
E J\left(1+\frac{G_{s}}{\kappa G A}\right) \frac{\partial^{4} w}{\partial x^{4}}-\left[\rho J\left(1+\frac{E}{\kappa G}+\frac{G_{s}}{\kappa G A}\right)+m_{s} \frac{E J}{\kappa G A}\right] \frac{\partial^{4} w}{\partial x^{2} \partial t^{2}}+ \\
+\frac{\rho J}{\kappa G}\left(\rho+\frac{m_{s}}{A}\right) \frac{\partial^{4} w}{\partial t^{4}}-\left(\frac{E J k_{s}}{\kappa G A}+G_{s}\right) \frac{\partial^{2} w}{\partial x^{2}}+\left(\rho A+\frac{k_{s} \rho J}{\kappa G A}+m_{s}\right) \frac{\partial^{2} w}{\partial t^{2}}+k_{s} w= \\
=\left(1-\frac{E J}{\kappa G A} \frac{\partial^{2}}{\partial x^{2}}+\frac{\rho J}{\kappa G A} \frac{\partial^{2}}{\partial t^{2}}\right) P \delta(x-v t) .
\end{gathered}
$$

Equation (4) describes vibrations of Timoshenko beam on three-parameter inertial foundation under the moving force.

\section{Solution of the problem}

In the case of simply supported beam solution of equation (4), describing the transverse vibrations of the undamped system, can be written as

$$
\begin{aligned}
w(x, t)= & \sum_{n=1}^{\infty}\left(C_{1 n} \cos \omega_{1 n} t+C_{2 n} \sin \omega_{1 n} t+C_{3 n} \cos \omega_{2 n} t+\right. \\
& \left.+C_{4 n} \sin \omega_{2 n} t+K_{1 n} \sin \frac{n \pi v t}{l}\right) \sin \frac{n \pi x}{l},
\end{aligned}
$$

where $\omega_{1 n}$ and $\omega_{2 n}$ are natural frequencies of the beam:

$$
\omega_{1 n}=\left[\frac{1}{2}\left(A_{1 n}-\sqrt{A_{1 n}^{2}-4 A_{2 n}}\right)\right]^{1 / 2}, \quad \omega_{2 n}=\left[\frac{1}{2}\left(A_{1 n}+\sqrt{A_{1 n}^{2}-4 A_{2 n}}\right)\right]^{1 / 2},
$$

and

$$
\begin{aligned}
& \alpha_{n}=\frac{n \pi}{l}, K_{1 n}=\frac{2 P\left(S_{1 n}+S_{2 n}\right)}{l\left(S_{3 n}-S_{4 n}+S_{5 n}\right)}, S_{1 n}=\kappa G A, S_{2 n}=J \alpha_{n}^{2}\left(E-\rho v^{2}\right) \text {, } \\
& S_{3 n}=E J \alpha_{n}^{2}\left(k_{s}+G_{s} \alpha_{n}^{2}\right)+\kappa G A\left(k_{s}+G_{s} \alpha_{n}^{2}+E J \alpha_{n}^{4}\right), \\
& S_{4 n}=v^{2} \alpha_{n}^{2}\left\{\kappa G A\left(m_{s}+\rho A\right)+\rho J k_{s}+J\left[E\left(m_{s}+\rho A\right)+\rho G_{s}+\rho \kappa G A\right] \alpha_{n}^{2}\right\} \text {, } \\
& S_{5 n}=v^{4} \alpha_{n}^{4} J \rho\left(m_{s}+\rho A\right) \text {, } \\
& A_{1 n}=\frac{1}{\rho}\left[\left(E+\frac{\rho G_{s}+\kappa G A \rho}{m_{s}+\rho A}\right) \alpha_{n}^{2}+\frac{\kappa G A}{J}+\frac{\rho k_{s}}{m_{s}+\rho A}\right], \\
& A_{2 n}=\frac{1}{\rho\left(m_{s}+\rho A\right)}\left[E\left(\kappa G A+G_{s}\right) \alpha_{n}^{4}+\left(E k_{s}+\frac{\kappa G A G_{s}}{J}\right) \alpha_{n}^{2}+\frac{\kappa G A k_{s}}{J}\right] \text {. }
\end{aligned}
$$

Constants $C_{1 n}, C_{2 n}, C_{3 n}$ and $C_{4 n}$ in expression (5) must be calculated from initial conditions of the problem: 


$$
\begin{gathered}
\left.w(x, t)\right|_{t=0}=0,\left.\quad \frac{\partial w(x, t)}{\partial t}\right|_{t=0}=0 \\
\left.\frac{\partial^{2} w(x, t)}{\partial t^{2}}\right|_{t=0}=0,\left.\quad \frac{\partial^{3} w(x, t)}{\partial t^{3}}\right|_{t=0}=\frac{2 P \alpha_{n} v}{\rho A l} .
\end{gathered}
$$

Taking into consideration (8), solution of equation (4) is given by

$$
\begin{gathered}
w(x, t)=\sum_{n=1}^{\infty}\left[-v \alpha_{n} \frac{2 P+K_{1 n} A l \rho\left(v^{2} \alpha_{n}^{2}-\omega_{2 n}^{2}\right)}{A l \rho \omega_{1 n}\left(\omega_{1 n}^{2}-\omega_{2 n}^{2}\right)} \sin \omega_{1 n} t+\right. \\
\left.+v \alpha_{n} \frac{2 P+K_{1 n} A l \rho\left(v^{2} \alpha_{n}^{2}-\omega_{1 n}^{2}\right)}{A l \rho \omega_{2 n}\left(\omega_{1 n}^{2}-\omega_{2, n}^{2}\right)} \sin \omega_{2 n} t+K_{1 n} \sin \alpha_{n} v t\right] \sin \alpha_{n} x,
\end{gathered}
$$

where $K_{1 n}$ is defined by formula (7).

Expression (9) describes forced vibrations of Timoshenko beam under concentrated load moving with constant velocity c, and is valid for $t \leq \frac{l}{v}$.

After the moving force descending the beam, free vibrations of the beam must be considered. In case of free vibrations $\left(t>\frac{l}{v}\right)$ solution for analysed beam can be written by

$$
\begin{gathered}
\tilde{w}(x, t)=\sum_{n=1}^{\infty}\left(\tilde{C}_{1 n} \cos \omega_{1 n} t+\tilde{C}_{2 n} \sin \omega_{1 n} t+\tilde{C}_{3 n} \cos \omega_{2 n} t+\right. \\
\left.+\tilde{C}_{4 n} \sin \omega_{2 n} t\right) \sin \frac{n \pi x}{l}
\end{gathered}
$$

$\tilde{C}_{1 n}, \tilde{C}_{2 n}, \tilde{C}_{3 n}$ and $\tilde{C}_{4 n}$ in expression (10) should be calculated taking into account initial conditions for free vibrations of the beam (for $t=l / v$ ):

$$
\begin{gathered}
\left.w\left(\frac{l}{2}, t\right)\right|_{t=\frac{l}{v}}=\left.\tilde{w}\left(\frac{l}{2}, t\right)\right|_{t=\frac{l}{v}},\left.\quad \frac{d}{d t} w\left(\frac{l}{2}, t\right)\right|_{t=\frac{l}{v}}=\left.\frac{d}{d t} \tilde{w}\left(\frac{l}{2}, t\right)\right|_{t=\frac{l}{v}}, \\
\left.\frac{d^{2}}{d t^{2}} w\left(\frac{l}{2}, t\right)\right|_{t=\frac{l}{v}}=\left.\frac{d^{2}}{d t^{2}} \tilde{w}\left(\frac{l}{2}, t\right)\right|_{t=\frac{l}{v}},\left.\quad \frac{d^{3}}{d t^{3}} w\left(\frac{l}{2}, t\right)\right|_{t=\frac{l}{v}}=\left.\frac{d^{3}}{d t^{3}} \tilde{w}\left(\frac{l}{2}, t\right)\right|_{t=\frac{l}{v}} .
\end{gathered}
$$

When consider damped vibrations of Timoshenko beam the equation of motion may be written as follows 


$$
\begin{gathered}
E J\left(1+\frac{G_{s}}{\kappa G A}\right) \frac{\partial^{4} w}{\partial x^{4}}-\left[\rho J\left(1+\frac{E}{\kappa G}+\frac{G_{s}}{\kappa G A}\right)+m_{s} \frac{E J}{\kappa G A}\right] \frac{\partial^{4} w}{\partial x^{2} \partial t^{2}}+ \\
+\frac{\rho J}{\kappa G}\left(\rho+\frac{m_{s}}{A}\right) \frac{\partial^{4} w}{\partial t^{4}}-\frac{E J c}{\kappa G A} \frac{\partial^{3} w}{\partial x^{2} \partial t}+\frac{\rho J c}{\kappa G A} \frac{\partial^{3} w}{\partial t^{3}}-\left(\frac{E J k_{s}}{\kappa G A}+G_{s}\right) \frac{\partial^{2} w}{\partial x^{2}}+ \\
\left(\rho A+\frac{k_{s} \rho J}{\kappa G A}+m_{s}\right) \frac{\partial^{2} w}{\partial t^{2}}+c \frac{\partial w}{\partial t}+k_{s} w=\left(1-\frac{E J}{\kappa G A} \frac{\partial^{2}}{\partial x^{2}}+\frac{\rho J}{\kappa G A} \frac{\partial^{2}}{\partial t^{2}}\right) P \delta(x-v t),
\end{gathered}
$$

where $c$ is a damping coefficient.

The method of solving the problem is the same as in the case of undamped vibrations, presented above in the paper.

\section{Numerical example}

Equations of vibrations of the beam are solved using Mathematica code. Two cases are investigated in the paper. The first case when the moving force is on the beam $\left(t \leq \frac{l}{v}\right)$, and the second one after the force descending the beam $\left(t>\frac{l}{v}\right)$.

Parameters of foundation are determined according to the Vlasov model [18], and are calculated for the soil of properties:

$$
\begin{aligned}
& E_{s}=100 \mathrm{MPa}-\text { soil modulus of elasticity, } \\
& v_{s}=0.3-\text { Poisson's ratio, } \\
& H=1.5 \mathrm{~m}-\text { thickness of the soil layer, } \\
& \gamma=26 \frac{\mathrm{kN}}{\mathrm{m}^{3}}-\text { soil weight per unit volume. }
\end{aligned}
$$

Figures show dimensionless deflection of the beam midpoint. In the Figure 1 deflection of the beam midpoint for various velocities of the moving force is compared. Figure 2 shows influence of foundation parameters to the deflection of the beam in case $v=60 \frac{\mathrm{m}}{\mathrm{s}}$.

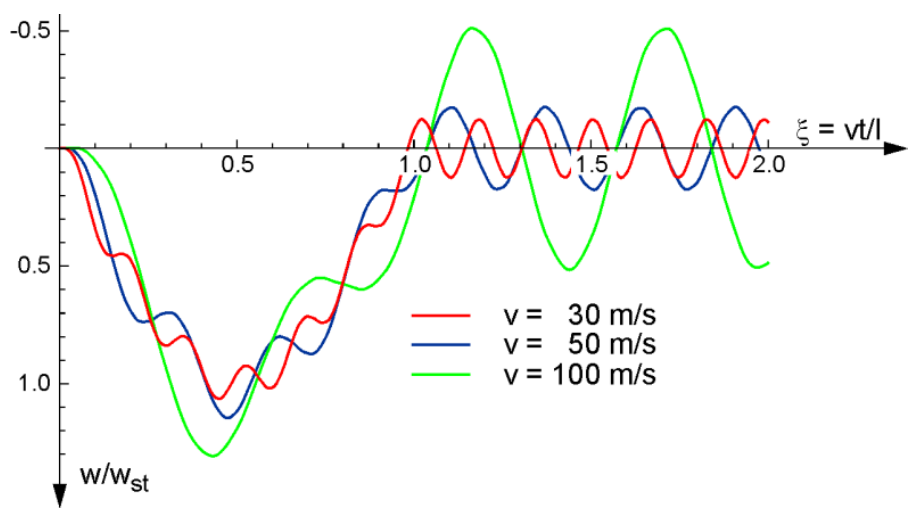

Fig. 1. Influence of velocity of the load for the midpoint deflections of Timoshenko beam on the Vlasov foundation. 


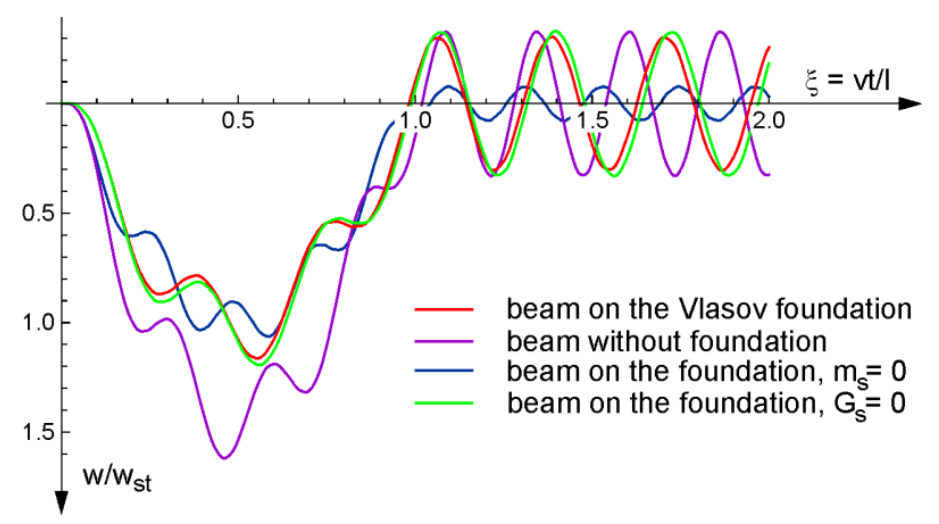

Fig. 2. Comparative analysis of Timoshenko beam midpoint deflections for various foundations, speed of the moving load $v=60 \frac{\mathrm{m}}{\mathrm{s}}$.

\section{Conclusion}

In the paper Timoshenko beam on three-parameter foundation under moving force is considered. The parameters take into account elasticity, shear and inertia of subgrade. Influence of these parameters to deflection of the beam is analysed. Influence of inertia of the foundation to the beam deflection is more significant than parameter $G_{s}$.

\section{References}

1. L. Frýba, Vibration of solids and structures under moving loads, (Thomas Telford, 1999)

2. L. Frýba, Dynamics of railway bridges, (Thomas Telford, London, 1996)

3. Y. B. Yang, J. D. Yau, Y. S. Wu, Vehicle-bridge interaction dynamics with applications to high-speed railway, (WS, Singapore, 2004)

4. S. P. Timoshenko, Philos. Mag., 41, 744 (1921)

5. S. P. Timoshenko, Philos. Mag., 43, 125 (1922)

6. W. Szcześniak, WUT Res. Pap., Constr., 112, 77, Warsaw (1990)

7. W. Szcześniak, Eng. Trans., 38, 591 (1990)

8. S. H. Crandall, Third Midwestern Conference on Solid Mechanics, Ann Arbor, Mich., 146 (1957)

9. S. Chonan, ZAMM, 59, 9 (1978)

10. R. Bogacz, S. Nowakowski, Acta Mech., 61, 117 (1986)

11. S. F. Felszeghy, J. Vib. Acoust., 118, 277 (1996)

12. E.Winkler, Die Lehre von der Elasticitaet und Festigeit, (Dominicus, Prag 1867)

13. H. P. Lee, Appl. Acoust., 55, 203 (1998)

14. V. I. Gulyaev, V. M. Mel'nik, E. V. Yakovenko, Int. Appl. Mech., 36, 1650 (2000)

15. P. L. Pasternak, GILSA, Moscow, (1954)

16. M. Radeş, Int. J. Solids Struct., 6, 739 (1970)

17. T. P. Nguyena, D. T. Phamb, P. H. Hoang, Science Direct, Proc. Eng. 142, 166 (2016)

18. V. Z. Vlasov, N. N. Leontev, (GIFML, Moscow, 1960) 